\title{
A case of type 1 muscle fibre hypotrophy and internal nuclei
}

\author{
T. INOKUCHI ${ }^{1}$, H. UMEZAKI, AND T. SANTA \\ From the Division of Neurology, Kyushu-Koseinenkin Hospital, Kitakyushu, and \\ the Department of Neurology, Neurological Institute, Kyushu University, Fukuoka, Japan
}

SYNOPSIS A 14 year old boy was diagnosed as suffering from type 1 muscle fibre hypotrophy with internal nuclei. On histological examination of a biopsied muscle, there was selective hypotrophy of type 1 muscle fibre with internal nuclei, and focal degenerative changes were seen in a few type 1 fibres. The small type 1 fibres were arranged in small or large groups in one bundle. An EMG study of moderately weak muscles revealed low amplitude and short duration motor unit potentials as well as normal potentials and no spontaneous discharges. The $\mathrm{H}$ reflexes were abnormally low in amplitude compared with the $\mathbf{M}$ response. The histological and electrophysiological findings suggested that the type 1 fibre involvement in the present case may have a neurogenic basis. It is likely that the clinical features of the reported cases are too variable for a single clinical entity.

A number of new types of congenital myopathies have been described in recent years. Engel et al. (1968) reported a case in which selective involvement of type 1 muscle fibres with a small diameter and central nuclei was found histochemically. They described it as "type 1 muscle fibre hypotrophy and central nuclei'. Engel and Devivo (1968) and Engel (1970) reported another case, and a similar case and two familial cases were reported by Brooke and Williamson (1969), and by Karpati et al. (1970), respectively. We have observed a very similar case.

The aetiology of this new type of muscular disorder is yet unknown, and it is still undetermined whether 'type 1 muscle fibre hypotrophy and central nuclei' is a clinical entity or not. The purpose of this report is to present a case of this rare type of muscular disorder and to compare its clinical characteristics with other reported cases.

\section{CASE REPORT}

The patient, a 14 year old boy, was admitted to our clinic on 17 February 1972. During his infancy, no

1 Present address: Department of Anatomy, Kurume University, Asahi-machi, 67, Kurume, 830-91, Japan.

(Accepted 10 December 1974.) remarkable changes were noticed by his parents except for relative deterioration in sucking activity. His illness was not progressive. His parents had not suffered from any muscular diseases.

On admission, the patient was $158 \mathrm{~cm}$ in stature, $44.5 \mathrm{~kg}$ in weight. He had macroglossia and a high arched palate. His head, mandible, and chest showed a mild deformity. He was mentally normal. Mild weakness and atrophy of the proximal muscles of the extremities were noted with winging of the scapula.

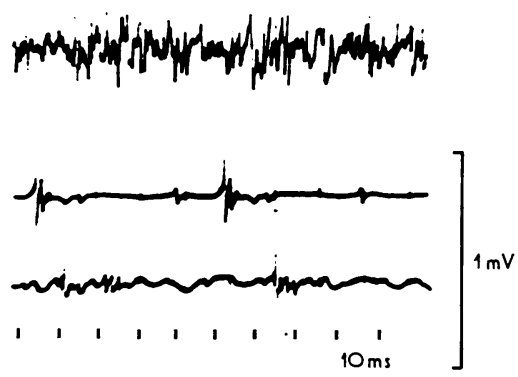

FIG. 1 Electromyographic volitional pattern in right biceps muscle. Upper record: moderate contraction. Middle and lower record: mild contraction. Pattern of low amplitude and short duration potentials is observed in the moderate contraction of the muscle. Polyphasic motor unit potentials are observed. Calibration, $1 \mathrm{mV}$. Time marker, $10 \mathrm{~ms}$. 


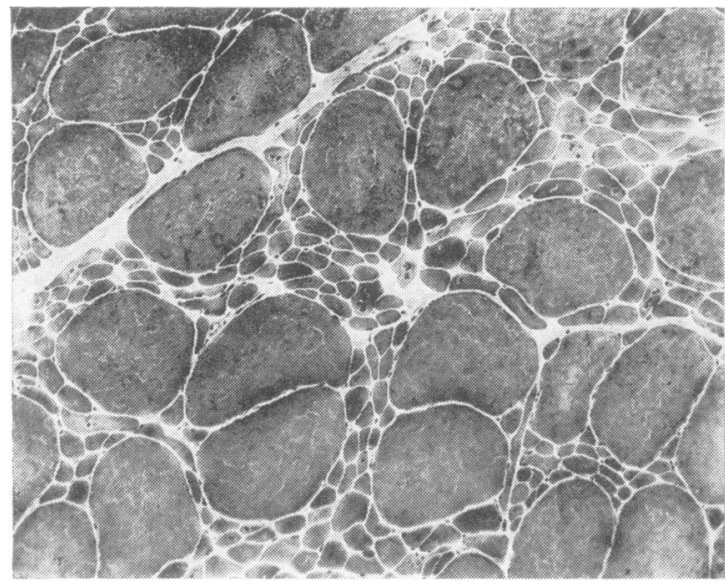

(a)

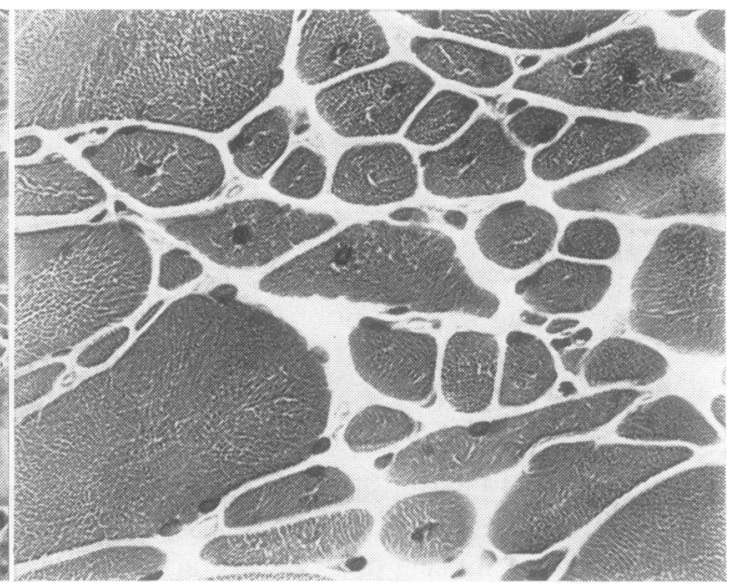

(b)

FIG. 2 (a) Cross-section of right biceps brachii muscle. The abnormally small fibres are distributed in small or large groups in a bundle. Haematoxylin and eosin, $\times 100$. (b) About one-third of the small fibres had internal nuclei (occasionally 'central'). The number of internal nucleus was usually single, but poly-nuclei were observed in a few fibres. $H$ and $E, \times 400$.

He showed a mild waddling gait and a positive Gowers' sign. All tendon reflexes of the extremities were absent and pathological reflexes were not elicited. There were no joint contractures. Myotonic phenomena and fasciculations were not detected. Coordination and sensation were normal. Laboratory studies revealed normal levels of serum enzymes

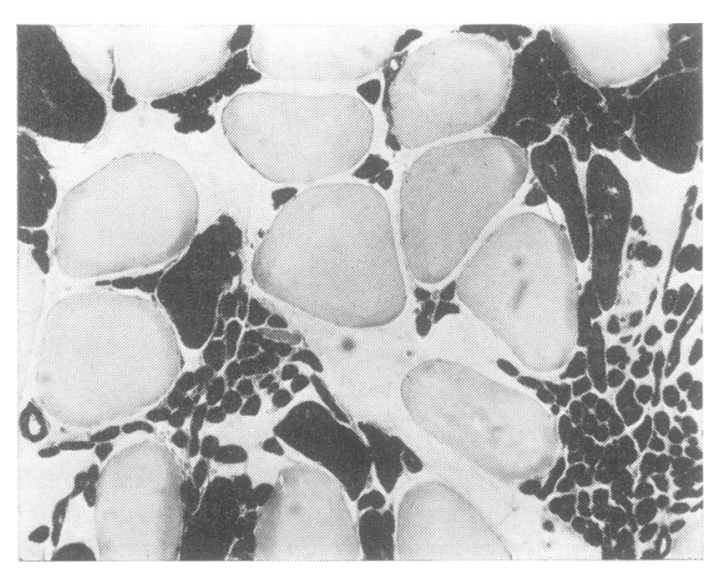

FIG. 3 Frozen section of the biceps brachii reacted for EDTA-activated myosin-ATPase. All of the small fibres are type 1 fibres histochemically. $\times 124$.

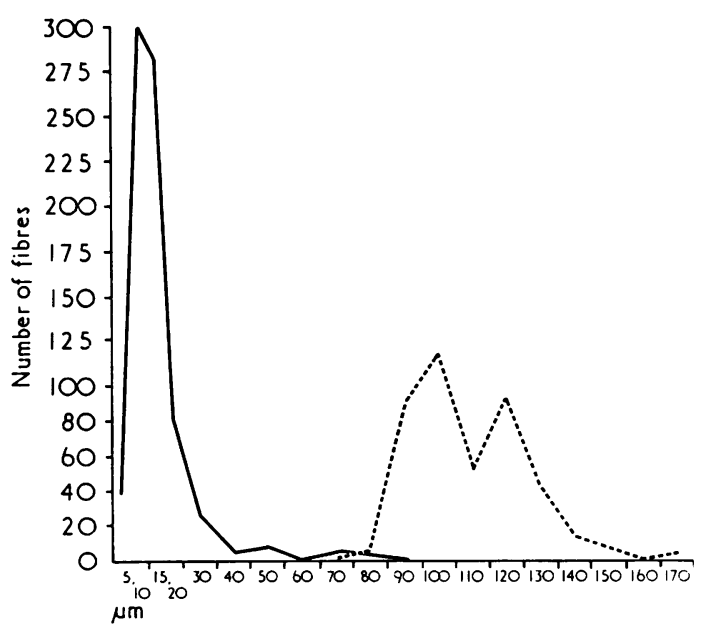

FIG. 4 Histogram of fibre diameters. Type 1 muscle fibres are very small for the most part, and are more numerous than type 2 fibres. - Type 1 (mean= $13 \mu \mathrm{m} . N=737)$. --- Type 2 (mean=114 $\mu \mathrm{m} . N=$ 424). 


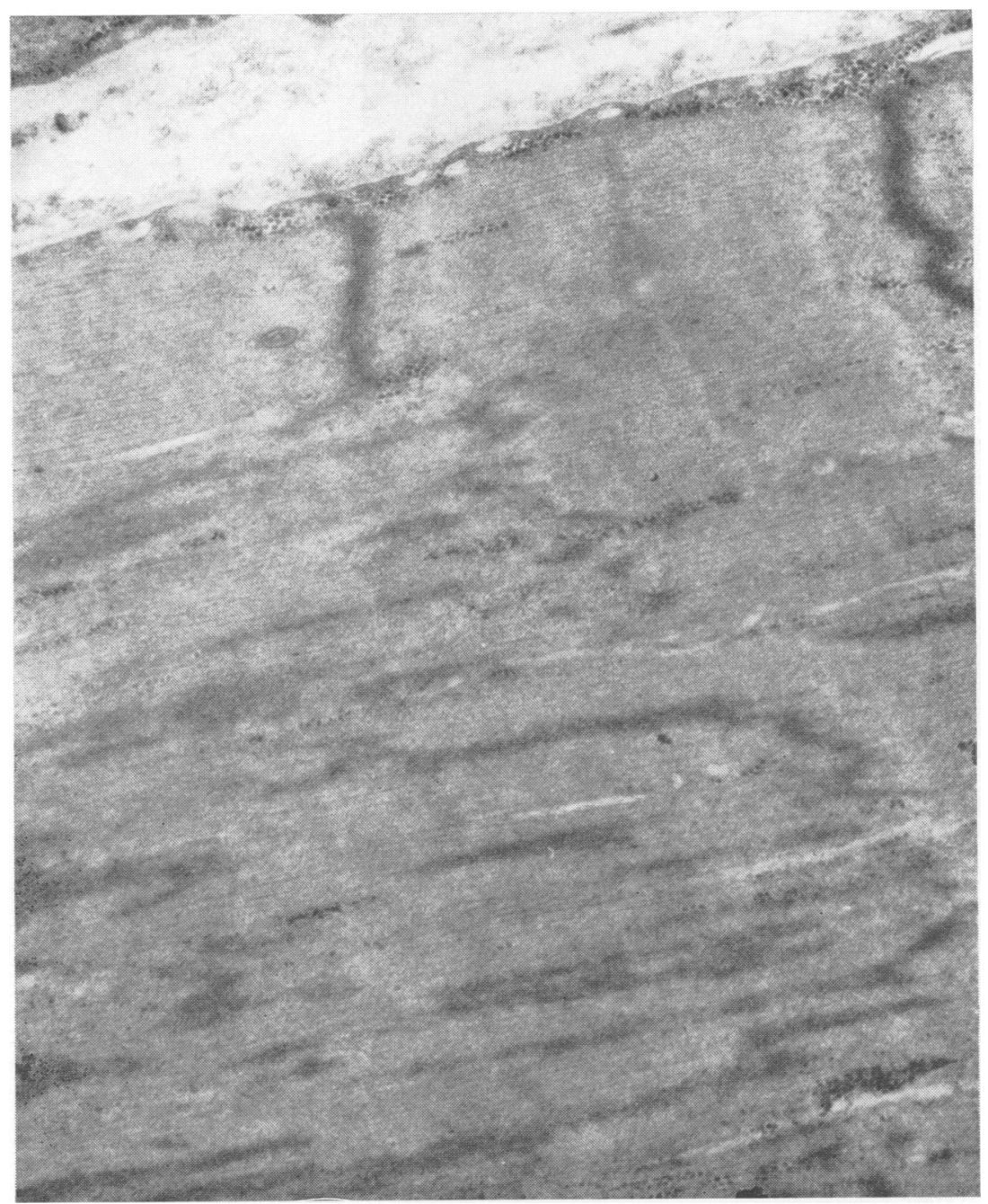

FIG. 5 Focal alteration of muscle fibre, illustrating $Z$-streaming with absence of mitochondria and disarrays of myofilaments. $\times 27000$.

(GOT, GPT, LDH, CPK). Total serum protein was $6.8 \mathrm{~g} / \mathrm{dl}$ with normal electrophoretic pattern. Quantitative immunoelectrophoresis of $\operatorname{IgA}, \operatorname{IgM}$, and IgG gave normal results. The ECG, vectorECG, and a phonocardiographic study showed no findings indicating the presence of latent cardiomyopathy. The CSF protein level was not elevated. Electromyographic (EMG) studies of proximal and distal muscles of the upper and lower limbs showed electrical silence at rest. The motor unit potentials were not decreased in number. Individual potentials were normal or short in duration, and normal or low in amplitude. Potentials of low amplitude and short duration were especially conspicuous in the brachial biceps muscles (Fig. 1). Polyphasic motor unit potentials of low amplitude were also present. Potentials with high amplitude and long duration were not observed. The $\mathrm{H}$ reflex of the lower limb was abnormally low in amplitude compared with the $\mathrm{M}$ response. Tetanization showed no waning or waxing phenomenon. Motor nerve conduction velocities were normal.

MUSCLE BIOPSY A small piece of the muscle biopsied from the right biceps brachii was fixed in $10 \%$ neutral formalin, embedded in paraffin, and stained with haematoxylin-eosin ( $\mathrm{H}$ and $\mathrm{E})$, periodic acid Schiff (PAS), PTAH, and by Gomori's trichrome stain. The second portion was prepared for frozen sections, stained with $\mathrm{H}$ and $\mathrm{E}, \mathrm{PAS}$, and reacted for 


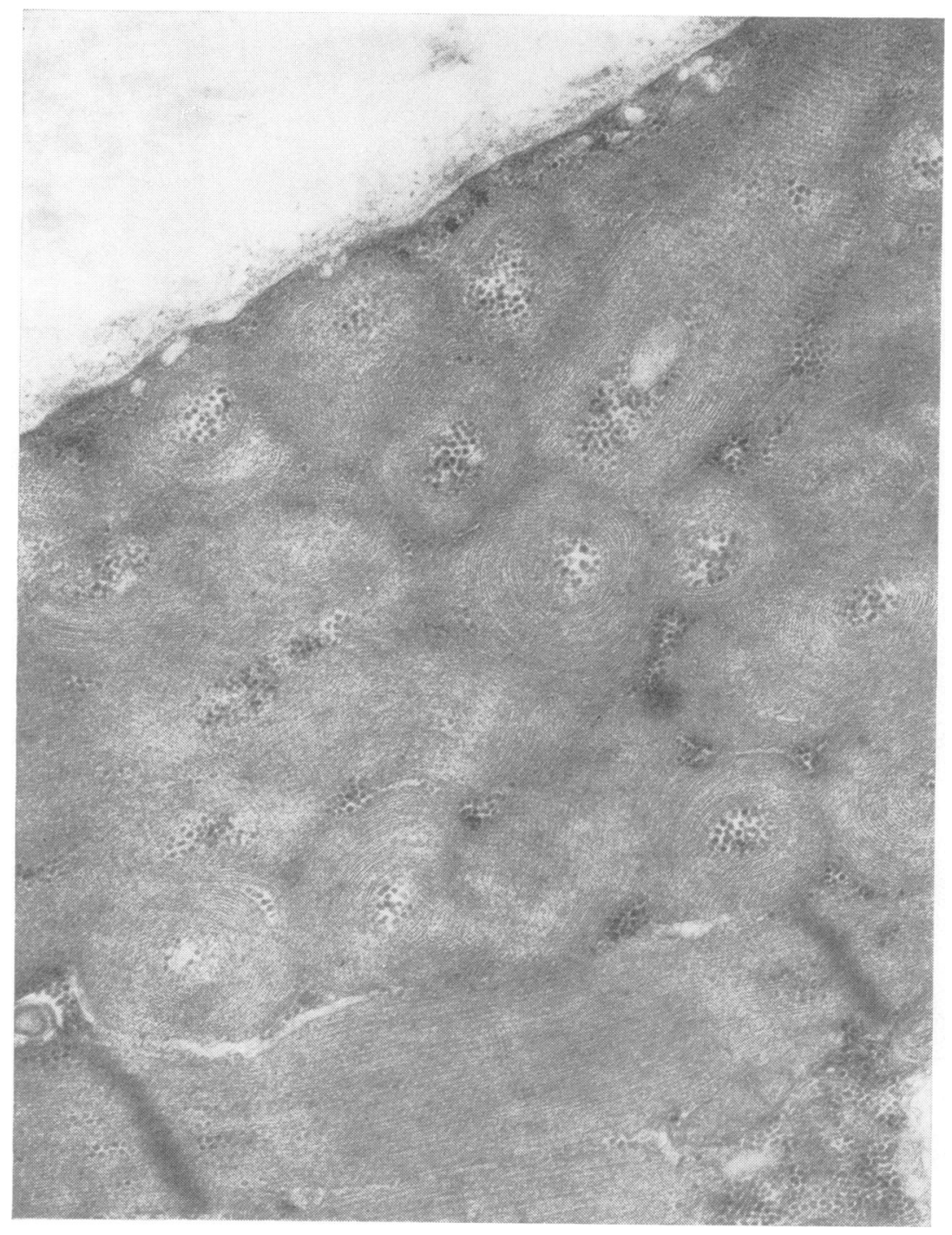

FIG. 6 One of the cytoplasmic inclusion bodies observed only rarely in the small fibres. Some cylindrical, filamentous bodies are accumulated in the subsarcolemmal region. $\times 34000$.

dihydronicotinamide adenine dinucleotide dehydrogenase (NADHD), myosin-ATPase (pH 4.5), and acid phosphatase activity. For electron microscopy, the tissue was fixed in 3\% glutaraldehyde buffered with $0.1 \mathrm{M}$ sodium cacodylate for three hours, and post-fixed in $1 \%$ osmium tetroxide for two hours, dehydrated in graded alcohols and embedded in Epon 812. Thin sections stained with uranyl acetate and lead acetate were examined with a JEM-7A electron microscope.

LIGHT MICROSCOPY In transverse sections there were two populations of muscle fibres according to their diameters. The small fibres were more numerous than the large ones, and were arranged in small or large groups within one bundle (Fig. 2). About one third of the small fibres had internal (occasionally 'central') nuclei (24.6-38.4\%, mean $31.4 \%$ ). The internal nuclei were usually single, but two or three nuclei were observed in a few fibres. The large fibres showed normal architecture. Basophilic fibres were not seen. There was no increase of endomysial connective tissue.

HISTOCHEMICAL STUDY The small fibres were all of type 1 histochemically (Fig. 3), and the type 1 fibres 


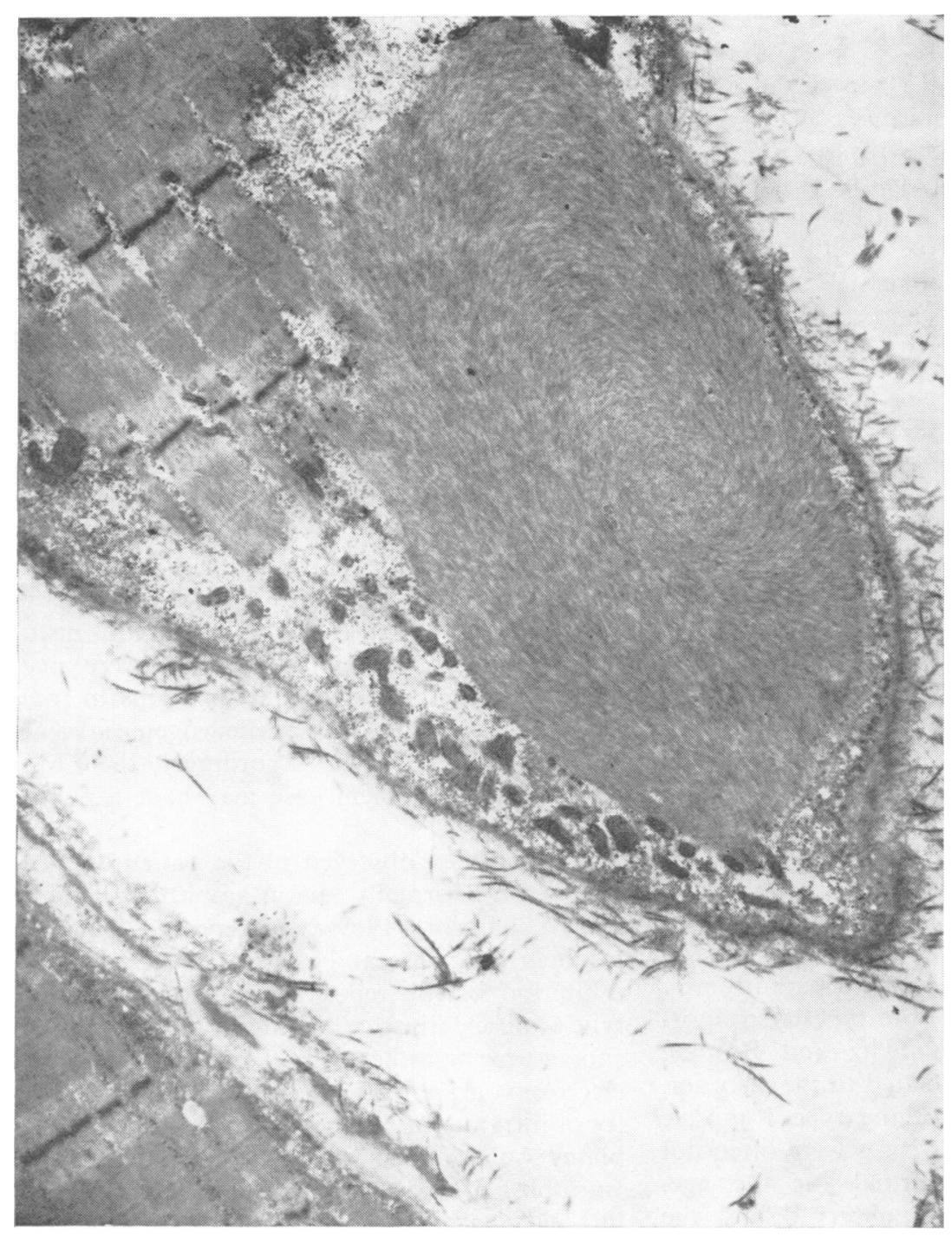

FIG. 7 Another type of cytoplasmic inclusion body, consisting of fine filamentous segments. $\times 10000$.

were generally very small. The diameter of type 1 fibres ranged from 3 to $80 \mu \mathrm{m}$, averaging $13 \mu \mathrm{m}$. The diameter of type 2 fibres ranged from 75 to $165 \mu \mathrm{m}$, averaging $114 \mu \mathrm{m}$ (larger than normal) (Fig. 4). All hypertrophic fibres were type 2 histochemically. With NADHD and ATPase reactions, some fibres displayed a lack of enzyme activity in their central regions.

ELECTRON MICROSCOPY The appearance of the internal nuclei was normal and there were no abnormal findings in the central or perinuclear regions of the cell. The mitochondria were normal. In a few fibres there was streaming of $Z$-discs and focal decrease in mitochondria where the myofilaments were disarrayed (Fig. 5). The large fibres showed no focal degeneration.

In addition, two kinds of cytoplasmic inclusions were observed in the small fibres. In the transverse section, one was large and oval, consisting of fine filamentous profiles (Fig. 6), and the other cylindrical, composed of 12 to 20 circular filaments arranged in ring-shaped structures (Fig. 7). 


\section{DISCUSSION}

Selective smallness of type 1 muscle fibre may occur in Werdnig-Hoffmann disease (Engel and Brooke, 1966), myotonic dystrophy (Engel and Brooke, 1966), nemaline myopathy (Engel et al., 1964; Gonatas et al., 1966; Martin and Reniers, 1968; Fardeau et al., 1970), myotubular myopathy (Dubowitz and Brooke, 1973a), 'type 1 muscle fibre hypotrophy and central nuclei' (Engel et al., 1968), and in tenotomized muscle (Engel et al., 1966).

The abnormalities revealed by EMG in the present case were not diagnostic of spinal muscular atrophy. Histologically, the lack of hypertrophic type 1 fibre in this case is against the diagnosis of Werdnig-Hoffmann disease (Engel and Brooke, 1966). There was no clinical or electromyographic evidence of myotonia. The histochemical and electron microscopic studies showed no myotube-like structures, nemaline rods, mitochondrial abnormalities, or abnormal increase of glycogen granules. The biopsied muscle had not been subjected to tenotomy or other trauma. Accordingly, it is reasonable to assume that the disease in the present case is most similar to the type 1 muscle fibre hypotrophy and central nuclei described by Engel et al. (1968).

In 1971, Brooke described an apparently new clinical entity 'congenital fibre type dysproportion' (Brooke, 1973; Dubowitz and Brooke, 1973b). Our case may be related to the disorder. But the mean diameters of the type 1 muscle fibres observed in Brooke's cases were often not significantly below the normal for the age. Internal nuclei were rarely observed and the clinical picture, such as the severity during the first two years of life, is different. Our case more closely resembles the previously mentioned nomenclature, type 1 muscle fibre hypotrophy and central nuclei.

An arrest of maturation of muscle was suspected in this disorder by previous authors (Engel et al., 1968; Brooke and Williamson, 1969; Engel, 1970). In the present case, most of the muscle fibres, whether small or not, showed normal architecture for the most part. These findings are in accord with observations by previous authors, and may support the above hypothesis. In addition, several small type 1 fibres contained multiple internal nuclei. With the electron microscope, Z-streaming and disarray of myofibrils were found in a few small fibres. Possibly the primary pathological change was selective hypotrophy of the type 1 fibre, and some of the hypotrophic fibres degenerated due to unknown cause.

The pathological significance of two kinds of cytoplasmic inclusions which were observed in this case is obscure. In the literature, these inclusion bodies have been observed in a limited number of unrelated diseases.

The EMG records in our patient suggest 'myopathy' in the general sense, but those EMG findings can be also observed with degeneration of scattered branches of the axonal tree, defective neuromuscular transmission or abnormalities of the motor neurone soma (Warmolts and Engel, 1970). As mentioned above, in histological studies most of the type 1 fibres were small and arranged in small or large groups in individual fascicles. This suggests neurogenic involvement of muscle fibres. Accordingly, the EMG findings in the present case may have a neurogenic basis.

The $\mathrm{H}$ reflex observed in the patient's lower limb was abnormally low in amplitude. Brooke and Williamson (1969) also reported the same result in their case and postulated an abnormal inhibition of the reflex system controlling the type 1 muscle fibres. The present patient had not shown any myasthenic phenomena clinically or electromyographically. A histological abnormality of intramuscular nerve fibres and motor endplates could not be looked for in the present case. In published reports on type 1 hypotrophy with internal nuclei, no morphological abnormalities of the peripheral nerves, motor end-plates, muscle spindles, and spinal cord have been described. More case studies and new histological and electromyographic techniques are needed to elucidate this intricate problem.

Another purpose of this report is to discuss the clinical features of type 1 muscle fibre hypotrophy and central nuclei. The common features of the six reported cases are as follows: (1) specific histological findings (although detailed findings differ from case to case); (2) onset in early infancy; (3) no involvement of facial muscles; (4) decreased tendon reflexes; (5) no mental deterioration; (6) no joint contrac- 
tures; (7) normal serum enzymes. Other clinical features are variable. Karpati et al. (1970) reported two familial cases, but the other cases occurred sporadically. Four of six cases showed a non-progressive course, but the remaining two showed slowly progressive weakness. A male patient described by Engel et al. (1968) suffered from severe muscular wasting and disturbances of respiration and swallowing in the neonatal period and died at $1 \frac{1}{2}$ years of age. An 11 year old girl had only very mild wasting. The beginning of walking was delayed in four cases, but not in one case. On electromyography, three cases showed a pattern of low amplitude and short duration motor unit potential, but two cases showed large and slow motor unit potentials coincidentally with the above. In one case reported by Engel et al. (1968), fibrillation potentials were recorded. Thus, the clinical features as a whole are not uniform.

Some cases of 'myotubular myopathy', or 'centronuclear myopathy' have also presented the selective smallness of type 1 muscle fibres described above. A case reported by Harriman and Haleem (1972) may be closely related to the present case but in their case $53 \%$ of the large cells (type 2 muscle fibres) also possessed internal nuclei. Bethlem et al. (1969) described a 16 year old girl who had suffered from a slowly progressive muscular wasting with cardiomyopathy. Muscle biopsy disclosed type 1 fibre atrophy and central nuclei in both fibre types. Farkas-Bargeton et al. (1968), Fardeau et al. (1970), Caille et al. (1971), and Prince et al. (1972) independently reported type 1 muscle fibre hypotrophy without central nuclei. Shafiq et al. (1972) reported a case of idiopathic cardiomyopathy with involvement of skeletal muscles and hypotrophy of type 1 fibres without central nuclei. Cancilla et al. (1971) described a familial myopathy with selective degeneration of type 1 muscle fibre. All of these cases may be related to each other.

As Harriman and Haleem (1972) have pointed out, 'centronuclear myopathy' is not a single clinical entity, but associated with different forms of genetic expression and varying patterns of muscle involvement. 'Myotubular myopathy', or 'centronuclear myopathy' and type 1 muscle fibre hypotrophy with central nuclei may represent not only clinically but also morphologically overlapping entities.

The author thanks Dr M. Murakami, Professor of the Department of Anatomy, Kurume University, for his helpful suggestions in the preparation of the manuscript.

\section{REFERENCES}

Bethlem, J., Wijngaarden, G. K. van, Meijer, A. E. F. H., and Hülsmann, W. C. (1969). Neuromuscular disease with type 1 fiber atrophy, central nuclei, and myotube-like structures. Neurology (Minneap.), 19, 705-710.

Brooke, M. H. (1973). Congenital fiber type dysproportion. In Clinical Studies in Myology, Part 2, pp. 147-159. Edited by B. A. Kakulas. International Congress Series No. 295. Excerpta Medica, Amsterdam.

Brooke, M. H., and Williamson, T. (1969). An adult case of type 1 muscle fiber hypotrophy: an abnormality of monosynaptic reflex function. (Abstract.) Neurology (Minneap.), 19, 280.

Caille, B., Fardeau, M., Harpey, J.-P., and Lafourcade, J. (1971). Hypotonie congénitale avec atteinte élective des fibres musculaires de type 1 . A propos de deux observations. Archives Françaises de Pédiatrie, 28, 205-220.

Cancilla, P. A., Kalyanaraman, K., Verity, M. A., Munsat, T., and Pearson, C. M. (1971). Familial myopathy with probable lysis of myofibrils in type 1 fibers. Neurology (Minneap.), 21, 579-585.

Dubowitz, V., and Brooke, M. H. (1973a). Definition of pathological changes seen in muscle biopsies. In Muscle Biopsy, pp. 78 and 278-279. Edited by V. Dubowitz and M. H. Brooke. Saunders: London.

Dubowitz, V., and Brooke, M. H. (1973b). The congenital myopathies. In Muscle Biopsy, pp. 28-288. Edited by V. Dubowitz and M. H. Brooke. Saunders: London.

Engel, W. K. (1970). Selective and nonselective susceptibility of muscle fiber types. A new approach to human neuromuscular diseases. Archives of Neurology (Chic.), 22, 97117.

Engel, W. K., and Brooke, M. H. (1966). Muscle biopsy as a clinical diagnostic aid. In Neurological Diagnostic Techniques, pp. 90-146. Edited by W. S. Fields. Thomas: Springfield, Ill.

Engel, W. K., Brooke, M. H., and Nelson, P. G. (1966). Histochemical studies of denervated or tenotomized cat muscle: illustrating difficulties in relating experimental animal conditions to human neuromuscular diseases. Annals of the New York Academy of Sciences, 138, 160-185.

Engel, W. K., and Devivo, D. (1968). Type 1 fiber hypotrophy and central nuclei: a non-fatal case. Read before the Washington-Philadelphia Neurological Society, 1968 (cited by Engel, K. (1970); Selective and nonselective susceptibility of muscle fiber types. A new approach to human neuromuscular diseases. Archives of Neurology (Chic.), 22, 97-117).

Engel, W. K., Gold, G. N., and Karpati, G. (1968). Type 1 fiber hypotrophy and central nuclei. A rare congenital muscle abnormality with a possible experimental model. Archives of Neurology (Chic.), 18, 435-444.

Engel, W. K., Wanko, T., and Fenichel, G. M. (1964). Nemaline myopathy: a second case. Archives of Neurology (Chic.), 11, 22-39.

Fardeau, M., Caille, B., Harpey, J. P., and Lafourcade, J. (1970). Etude histologique, histochimique et ultrastructurale de deux observations d'hypotonie congénitale avec atteinte sélective des fibres musculaires de type 1 
(hypotrophie simple dans un cas; hypotrophie et présence de bâtonnets dans le second cas). Revue Neurologique, 123, 61-62.

Farkas-Bargeton, E., Aicardi, J., Chevrie, J.-J., and Thieffry, S. (1968). Apport des techniques histoenzymologiques à l'étude des hypotonies congénitales. Revue Neurologique, 119, 513-524.

Gonatas, N. K., Shy, G. M., and Godfrey, E. H. (1966). Nemaline myopathy. The origin of nemaline structures. New England Journal of Medicine, 274, 535-539.

Harriman, D. G. F., and Haleem, M. A. (1972). Centronuclear myopathy in old age. Journal of Pathology, 108. 237-247.

Karpati, G., Carpenter, S., and Nelson, R. F. (1970). Type 1 muscle fibre atrophy and central nuclei. A rare familial neuromuscular disease. Journal of the Neurological Sciences, 10, 489-500.

Martin, L., and Reniers, J. (1968). Nemaline myopathy. 1. Histochemical study. Acta Neuropathologica, 11, 282-293.

Prince, A. D., Engel, W. K., and Warmolts, J. R. (1972). Type 1 myofiber smallness without central nuclei or myotonia. (Abstract.) Neurology (Minneap.), 22, 401.

Shafiq, S. A., Sande, M. A., Carruthers, R. R., Killip, T., and Milhorat, A. T. (1972). Skeletal muscle in idiopathic cardiomyopathy. Journal of the Neurological Sciences, 15, 303-320.

Warmolts, J. R., and Engel, W. K. (1970). A critique of the 'myopathic' electromyogram. Transactions of the American Neurological Association, 95, 173-174. 